\title{
1 Wie arbeiten Wissenschaftsjournalisten?
}

Journalisten und Wissenschaftler sind aufeinander angewiesen: Journalisten brauchen Geschichten und verlässliche Informationen, Wissenschaftler möchten ihre Arbeit und ihre Ergebnisse bekanntmachen und eine fundierte Einschätzung zu aktuellen Themen geben. Das gilt besonders, wenn ein Thema in die Schlagzeilen geraten ist. Nicht immer gelingt allerdings die Interaktion: Aus Sicht der Wissenschaftler werden oftmals nicht die richtigen Experten gefragt oder Aussagen verkürzt, verdreht oder falsch dargestellt. Aus Sicht der Journalisten antworten Wissenschaftler auf Anfragen oft nicht schnell oder nicht prägnant genug.

Damit Wissenschaftler ihre Botschaften besser vermitteln können, ist es hilfreich, wenn sie sich den Arbeitsalltag von Journalisten und die Anforderungen, die an sie gestellt werden, vor Augen führen. Beispielhaft berichten hier zwei Wissenschaftsjournalistinnen (Print, Radio) über ihre Arbeit. Beide hatten zusammen mit weiteren Journalisten im September 2011 an einem von der TMF organisierten Workshop teilgenommen, dessen Ziel es war, das gegenseitige Verständnis der Arbeitsweisen von Journalisten und Wissenschaftlern zu verbessern (s. http://www.tmf-ev.de/news/965). 


\subsection{Bei der Zeitung: Fragen an Christina Berndt (Süddeutsche Zeitung)}

\section{Wie sieht ein typischer Arbeitstag bei Ihnen aus?}

So ein Arbeitstag ist bei uns in viele Konferenzen gegliedert, sodass allein dadurch schon viel Zeit wegfällt. Man kommt so gegen halb neun zur Arbeit und verschafft sich erst mal einen Überblick über die Nachrichtenlage. Was beschäftigt uns heute, was sind die Themen? Dabei wirft man durchaus auch den Blick auch auf die politischen Themen, die ja auch Anlässe oder Hintergründe für die Wissenschaftsberichterstattung bieten können. Wir schnuppern also ein bisschen herum. Um halb zehn beginnt die erste interne Konferenz in den einzelnen Ressorts und um zehn Uhr die große Konferenz. Da sollte das Tagesprogramm der einzelnen Resorts dann eigentlich schon stehen. In der großen Konferenz wird zum Beispiel geschaut, dass man keine Doubletten hat. Manchmal wird etwas gebündelt. So ein riesiges Thema wie Flüchtlinge beispielsweise will man nicht an fünf Stellen im Blatt haben, sondern das wird gebündelt an zentraler Stelle gemacht. Es kann auch mal sein, dass die Seite zwei auf ein Thema zugreift, wenn es von übergeordneter Bedeutung ist. Das war neulich so als die Gravitationswellen Schlagzeilen gemacht haben. Dann gibt es nachmittags, so um 17 Uhr, nach dem Andruck, noch eine Konferenz, wo auch in die nächsten Tage geschaut wird.

Die Aufgaben zwischendurch sind ganz unterschiedlich. An manchen Tagen ist man Autor, an anderen Tagen produziert man die Seiten. Da gibt man dann Beiträge in Auftrag, entweder an Kollegen intern, auch an Korrespondenten irgendwo in der Welt, oder an Freie, die auch selber Themen anbieten. Wir schreiben nicht immer tagesaktuell, aber wenn so etwas wie die Nachricht von den Gravitationswellen kommt, dann muss das aktuell ins Blatt und dann muss das zwischen morgens neun und abends 17 Uhr stehen, und zwar druckreif. Das ist natürlich anders als in der Wissenschaft, wo eine Publikation über einen viel längeren Zeitraum vorbereitet wird. Bei uns muss das fertig sein, und zwar auf die Minute. Da wartet niemand.

Die erste Auflage, die ins Ausland geht, wird schon so ab halb fünf gedruckt, gerade auch die Wissenschaft, der Rest erst so um 17.15 Uhr. Anschließend können wir weiter updaten. Die Seiten der Wissenschaft werden allerdings gemeinhin nicht grundlegend überarbeitet, man holt Fehler raus, wenn es vorher ziemlich knapp war. Aber eigentlich bleiben die Seiten dann wie sie sind. In den aktuellen Ressorts dagegen kann natürlich die Zeitung, die um 17 Uhr, und die, die um 19 Uhr gedruckt wird, komplett unterschiedlich aussehen, wenn akut etwas Wichtiges passiert.

Man arbeitet aber nicht immer unter Hochdruck. Ich habe auch langfristigere Themen, gerade auch investigative Recherchen. Da habe ich ewig Zeit. Manchmal ist das im Tagesgeschäft allerdings auch schwierig, und für wichtige Stücke hängt man dann auch die Nächte dran. 


\section{Wie gehen Sie vor, wenn Sie Themen suchen?}

Das ist meist eine wilde Mischung aus E-Mails, Fremdpresse und Pressemitteilungen. Man muss durchaus aber auch die Nase in den Wind halten. Manchmal gibt es eine ganz eigene Themengenese - bis hin dazu, dass einen die Nachbarin im Hausflur irgendetwas fragt und man denkt, ja, das bewegt die Leute, das könnte man doch einmal machen.

\section{Was macht ein Thema zum Thema?}

Journalistisch ist natürlich immer ein Anlass gut. Also: Gibt es eine Neuigkeit dazu? Womöglich eine wissenschaftliche Publikation? Aber natürlich auch, ob sich in der Weltgeschichte gerade irgendetwas entwickelt. Wenn politisch so viel passiert, wie jetzt beim Zika-Virus, dann ist das natürlich aktuell. Das Virus gibt es zwar schon ein paar Jahre, aber jetzt hat es sich dramatisiert bis hin zu Aufrufen, nicht schwanger zu werden. So etwas ist natürlich ganz akut ein Thema. Aber es können auch Themen sein, die eher eine gefühlte Aktualität haben. Man hat vielleicht den Gedanken, dass man jetzt mal was zur Pränataldiagnostik machen müsste. Ein Anlass ist auch, wenn es einen Protagonisten gibt, wenn man beispielsweise eine Person hat, die in der heutigen Zeit an Tuberkulose erkrankt ist und die eine sehr langwierige Infektion hat, die man nicht in den Griff kriegt. Dann kann man so eine Geschichte auch an einem Menschen erzählen. Damit hat sie gleich eine ganz andere Aktualität und eine ganz andere Nähe, als wenn ich eine wissenschaftliche Fachpublikation als Ausgangspunkt habe. Das kann durchaus eine Geschichte von einer ganz normalen Person sein. Aber natürlich machen auch Prominente Themen zu Themen. Als zum Beispiel Johannes Rau ein Aneurysma hatte und operiert wurde, hat sich plötzlich ganz Deutschland für Aneurysmen interessiert. Oder die Kanzlerin, als sie an Krücken ging, da habe ich zum Beispiel etwas über Hüftbrüche gemacht, weil dann die Aufmerksamkeit dafür vorhanden ist.

\section{Was macht für Sie einen guten Gesprächspartner aus?}

Bei einem Gesprächspartner suche ich zuerst eine Einordnung. Bei vielen Themen habe ich selbst schon einen gewissen Expertenstatus erreicht, da kann ich auch aus dem Ärmel vieles schreiben, aber das ist natürlich nicht das übliche journalistische Vorgehen. Bei anderen Themen bin ich auch völlig unbeleckt. Ich suche mir also Leute, die mehr wissen als ich. Und die suche ich mir oft anhand von Fachpublikationen. Denn wenn ich zum Beispiel über eine Traumabehandlung nach einem schweren Zugunglück schreibe, dann möchte ich nicht mit irgendeinem Psychologen sprechen, der sonst vielleicht Familientherapie macht, sondern dann möchte ich natürlich den möglichst an einer Universität agierenden Fachmann für solche Traumata. Das ist mir ganz wichtig. Das muss ein richtiger Experte auf diesem Teilge- 
biet sein. Ich will auch nicht irgendeinen Virologen, wenn ich über das ZikaVirus schreibe, so wie ich das in meinen Anfängen auch noch gemacht habe. Sondern ich möchte jemanden, der sich genau mit der Gattung der Flaviviren auskennt. Oder wenn es um Ebola geht, dann möchte ich nicht einen Grippeforscher, sondern da möchte ich den Fachmann, der diese Viren kennt. Oder den Praktiker von Ärzte ohne Grenzen, der da gerade sechs Monate war. Das ist ein guter Experte.

Natürlich kommt es auf das Medium an, was ist ein guter Experte ist. Ich bei der Zeitung bin da relativ anspruchslos. Wenn jemand mit mir halbwegs so reden kann, dass ich ihn verstehe, dann reicht mir das schon. Für das Fernsehen ist ein guter Experte dagegen unbedingt jemand, der möglichst unfallfrei in eine Kamera spricht. Das sind ganz andere Anforderungen, weshalb im Fernsehen leider immer dieselben Leute auftauchen. Bei der Zeitung kann ich ja den Wissenschaftlern sozusagen helfen, ihre Aussagen zu formulieren, indem ich eben nicht seitenweise O-Töne zitiere, sondern die Inhalte ausdrücke und nur zwischendrin dann mal ein Zitat des Wissenschaftlers einfließen lasse. Trotzdem bin ich natürlich froh über jeden, der das so gut rüberbringt, dass ich nicht mehr viel übersetzen muss. Der es auch schafft, ein bisschen Abstand von seiner Arbeit zu nehmen und sich nicht in zu vielen Details zu verlieren, die vielleicht sein Steckenpferd sind. Das ist oft schmerzlich für Wissenschaftler, da sie so viel Energie in diese Detailforschung stecken und die so wichtig ist. Aber für den allgemeinen Leser sind die Details eben dann doch nicht wichtig. Das ist auch eine Übungssache. Ich finde es toll, wenn Wissenschaftler diese Vermittlung versuchen und immer mal trainieren. Es geht ja damit los, dass sie einfach ihren Kindern von ihrer Arbeit erzählen oder den Freunden der Kinder.

\section{Zur Person}

Dr. Christina Berndt beschäftigt sich bei der „Süddeutschen Zeitung“ mit dem Themenbereich Medizin und Lebenswissenschaften. Sie studierte Biochemie mit dem erklärten Ziel, Wissenschaftsjournalistin zu werden. Zunächst arbeitete sie wissenschaftlich und wurde für ihre Doktorarbeit am Deutschen Krebsforschungszentrum mit dem Promotionspreis der Deutschen Gesellschaft für Immunologie ausgezeichnet. Schon währenddessen schrieb sie für die „RheinNeckar-Zeitung "über Medizin und Forschung. Es folgten Praktika bei der Deutschen Presseagentur, dem Süddeutschen Rundfunk, dem „Spiegel“, „Bild der Wissenschaft“ und der „Süddeutschen Zeitung“, zu deren Redaktion sie seit März 2000 gehört. Sie erhielt zahlreiche Journalistenpreise (2006: European Science Writers Award, 2013: Wächterpreis der Tagespresse) und Nominierungen. Unter anderem ist sie auch Autorin des Bestsellers „Resilienz - Das Geheimnis der psychischen Widerstandskraft" (dtv premium). 


\subsection{Für das Radio: Fragen an Christina Sartori (freie Wissenschaftsjournalistin)}

\section{Wie sieht ein typischer Arbeitstag bei Ihnen aus?}

Die meisten meiner Aufträge als freie Wissenschaftsjournalistin betreffen tagesaktuelle Themen: Ich erhalte manchmal am Nachmittag vorher, häufiger aber erst am Morgen oder Vormittag des betreffenden Tages einen Anruf mit zwei Informationen: 1. Das Thema, zu dem ich recherchieren soll, und 2. die Uhrzeit, zu der ich live in die Sendung geschaltet werde. Als erstes suche und buche ich in Berlin beim ARD Hauptstadtstudio oder dem Deutschlandradio eine Studiozeit, um nachmittags von dort in bester Qualität zum Beispiel ins Kölner Sendestudio des WDR zugeschaltet zu werden. Dann versuche ich, einen kompetenten Gesprächspartner zu finden - und vor allem: ihn persönlich zu sprechen. Um Fakten, die ich von einer früheren Recherche her kenne oder die ich gelesen habe, bestätigt zu bekommen. Um Zusammenhänge $z u$ verstehen. Um eine Einordnung von einem Experten zu erhalten. Um ein oder mehrere Zitate bekommen.

Erst wenn meine Recherche abgeschlossen ist, kann ich entscheiden, welche Richtung das Gespräch nehmen soll: Was ist die entscheidende Botschaft? Wie beginnt und endet das Gespräch? Habe ich das entschieden, schreibe ich einen Gesprächsleitfaden: Einen kompletten Gesprächsablauf, mit Anmoderationsvorschlag, Fragen für den Moderator und mit den Antworten, die ich geben werde. Diesen Gesprächsablauf erhält der Redakteur. Im besten Falle kann der Redakteur den Gesprächsvorschlag sofort, nachdem er ihn erhalten hat, gegenlesen, manchmal sitzt er aber gerade in einer Konferenz oder bespricht gerade einen anderen Beitrag für die Sendung. Wenn ich die Rückmeldung erhalte, füge ich gewünschte Änderungen ein oder diskutiere mit dem Redakteur über mögliche und unmögliche Änderungen. Den endgültigen Gesprächsleitfaden sollte der Moderator mindestens zwei Stunden vor Beginn der Sendung erhalten, um das Gespräch in die Sendung einbauen zu können. Notfalls reicht auch eine halbe Stunde vor Sendebeginn, aber nur, wenn gute Gründe vorliegen. Schließlich kann der Moderator sich nicht auf alle Themen erst in der letzten halbe Stunde vor Sendebeginn vorbereiten.

Nicht selten fragen mehrere Sender gleichzeitig bei mir an: Der eine möchte das Thema in einem Fünf-Minuten-Gespräch in seiner Sendung haben, der andere macht nur Beiträge, die maximal 2'30 lang sind. Oder ein anderer Sender möchte einen Kommentar. Dementsprechend weniger Zeit habe ich für die Recherche, weil ich verschiedene Gespräche oder Beitragsformen schreiben und besprechen muss.

Als ich Redakteurin beim WDR war, musste ich morgens prüfen, welche Themen in der heutigen Sendung meiner Meinung nach vorkommen sollten. In der morgendlichen Redaktionskonferenz diskutierten alle Kollegen 
gemeinsam, welche Themen in die Sendung gehören und in welcher Form: Gespräch, gebauter Beitrag, Kommentar, O-Ton-Schnipsel mit begleitendem Internettext. Anschließend mussten entsprechend freie Autoren beauftragt werden, oder ich recherchierte selber, um das Thema später selber „auf den Sender zu bringen“. Die Reihenfolge der Themen muss festgelegt werden, der Moderator erhält eine erste Übersicht über den Ablauf der Sendung und eventuelle Besonderheiten, z.B. die Voraufzeichnung eines Gesprächs etc. Teasertexte für das Internet müssen geschrieben, Gesprächsleitfäden gelesen und besprochen werden. Es ist wichtig, den Moderator stets auf dem Laufenden zu halten und regelmäßig die Nachrichtenlage zu prüfen: Falls am frühen Nachmittag ein sehr wichtiges neues Thema auftaucht, muss die Sendung umgeplant werden. Zwischendrin werden außerdem Themen für den nächsten Tag angedacht oder Beiträge bearbeitet, die längerfristig geplant sind.

Etwa eine halbe Stunde vor Sendebeginn geht der Senderedakteur mit dem Moderator ins Studio, um dort die letzten Vorbereitungen zu treffen, Studiogäste zu begrüßen, etc. Während der Sendung achtet der Redakteur auf Inhalt und Länge der Gespräche, korrigiert die minutengenaue Planung der Sendung, kontrolliert die Agenturen auf aktuelle Entwicklungen im Tagesgeschehen. Nach der Sendung trifft sich die Redaktion zu einer kurzen Sendekritik.

\section{Was macht ein Thema zum Thema?}

- dass viele Menschen davon betroffen sind (Erkältungswelle)

- dass alle darüber reden (Zika-Virus, Fasten)

- dass es eine bahnbrechende Entdeckung ist (Gravitationswellen gemessen)

- dass es sehr umstritten ist (Impfung)

- dass viele Menschen darüber nur falsches Halbwissen besitzen (Pille danach)

- dass es ein Jahrestag etc ist (10 Jahre Vogelgrippe)

- dass es ein Prinzip verdeutlicht (Studie die zeigt, dass viele präklinische Versuche von sehr schlechter Qualität sind)

\section{Wie gehen Sie vor, wenn Sie Themen suchen?}

Ich lese verschiedene Wissensseiten von Tageszeitungen, habe mehrere Newsletter abonniert, besuche Pressekonferenzen, Kongresse etc. Oft erhalte ich auch bei Interviews zu einer Geschichte eine Anregung für ein weiteres Thema.

Und wenn zum Beispiel die Bild-Zeitung mit „Angelina Jolie lässt sich Brüste abnehmen" aufmacht, dann schlage ich möglicherweise vor, etwas über die Möglichkeiten und Grenzen der Vorhersage von familiärem Brustkrebs zu machen. 


\section{Was macht einen guten Interviewpartner aus?}

Dass er so schnell wie möglich zu sprechen ist und meine Fragen beantworten kann: Zusammenhänge erläutern, einordnen, eine Übersicht über die Studienlage geben. Schön sind kleine Anekdoten oder Beispiele, die einen Punkt veranschaulichen; hilfreich ist es, auch die negativen Aspekte zu nennen, „den Haken an der Sache“. Ich bin stets dankbar, wenn mir jemand mitten in seinem Berufsalltag fünf oder zehn Minuten lang meine Fragen beantwortet, denn sonst könnte ich meine Arbeit nicht machen. Mehr ist meistens nicht notwendig, denn allzu detaillierte Schilderungen oder „Mini-Vorträge“ machen das Verstehen in der Regel nicht einfacher.

\section{Zur Person}

Im Anschluss an ihr Studium der Biologie (Diplom) an der FU Berlin, der Duke University, USA und der Université de Rennes, Frankreich, studierte Christina Sartori Wissenschaftsjournalismus an der FU Berlin. Nach acht Jahren als Redakteurin der Wissenschaftsredaktion des WDR Hörfunks arbeitet sie seit 2010 als freie Journalistin für den öffentlich-rechtlichen Hörfunk, z.B. WDR und Deutschlandfunk. Außerdem moderiert sie Veranstaltungen zu wissenschaftlichen Themen. 